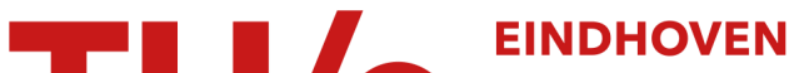

\section{Compensation for dynamic errors of coordinate measuring machines}

Citation for published version (APA):

Weekers, W. G., \& Schellekens, P. H. J. (1997). Compensation for dynamic errors of coordinate measuring machines. Measurement: Journal of the International Measurement Confederation, 20(3), 197-209.

https://doi.org/10.1016/S0263-2241(97)00032-8

DOI:

10.1016/S0263-2241(97)00032-8

Document status and date:

Published: 01/01/1997

\section{Document Version:}

Publisher's PDF, also known as Version of Record (includes final page, issue and volume numbers)

\section{Please check the document version of this publication:}

- A submitted manuscript is the version of the article upon submission and before peer-review. There can be important differences between the submitted version and the official published version of record. People interested in the research are advised to contact the author for the final version of the publication, or visit the $\mathrm{DOI}$ to the publisher's website.

- The final author version and the galley proof are versions of the publication after peer review.

- The final published version features the final layout of the paper including the volume, issue and page numbers.

Link to publication

\section{General rights}

Copyright and moral rights for the publications made accessible in the public portal are retained by the authors and/or other copyright owners and it is a condition of accessing publications that users recognise and abide by the legal requirements associated with these rights.

- Users may download and print one copy of any publication from the public portal for the purpose of private study or research.

- You may not further distribute the material or use it for any profit-making activity or commercial gain

- You may freely distribute the URL identifying the publication in the public portal.

If the publication is distributed under the terms of Article $25 \mathrm{fa}$ of the Dutch Copyright Act, indicated by the "Taverne" license above, please follow below link for the End User Agreement:

www.tue.nl/taverne

Take down policy

If you believe that this document breaches copyright please contact us at:

openaccess@tue.nl

providing details and we will investigate your claim. 


\title{
Compensation for dynamic errors of coordinate measuring machines
}

\author{
W. G. Weekers, P. H. J. Schellekens * \\ Eindhoven University of Technology, Precision Engineering Whal 1.25, Postbus 513, 5600 MB Eindhoven, The Netherlands
}

\begin{abstract}
Owing to the demand for shorter cycle times of measurement tasks, fast probing at coordinate measuring machines (CMMs) has become more important and therefore the influence of dynamic errors of CMMs will increase. This paper presents an assessment of dynamic errors owing to carriage motion, aimed at error compensation. In the adopted approach the major joint deflections as a result of accelerations are measured with position sensors. Other joint deflections are estimated based on analytical modelling of CMM components. Using a kinematic model of the CMM, the influences of the measured and estimated joint deflections on the probe position are calculated. The dynamic errors can be corrected by software compensation, based on the calculated values. The approach has been applied to an existing CMM, using inductive position sensors for on-line measurement of the major dynamic errors. Experiments show that the compensation method is very successful, enabling fast probing without serious degradation of measurement accuracy. (C) 1997 Elsevier Science Ltd.
\end{abstract}

Keywords: Coordinate measuring machine; Dynamic errors; Fast probing; Error modelling; Inductive position sensors; Error compensation

\section{Introduction}

Coordinate measuring machines (CMMs) are nowadays widely used for a large range of measurement tasks. These tasks are expected to be carried out with ever increasing accuracy, speed and flexibility, as well as the ability to operate under shop floor conditions. Research is necessary to meet these demands. Until recently, the research effort on improving CMM accuracy was mainly spent on quasi-static mechanical errors such as geometric errors, thermally induced errors and errors due to mechanical loads (mainly caused by the weight of moving parts). However, there are some trends concerning the use of CMMs that also make an assessment of the dynamic errors of CMMs which becomes increasingly important. These trends are:

- Increase in variety and complexity of measurement tasks. The often complex measurement

\footnotetext{
* Corresponding author.
}

tasks involve complex motion, making such tasks more prone to dynamic errors.

- Location of CMMs near the manufacturing process or even integration with production lines. The environmental conditions here, such as vibrations and thermal effects, result in errors and a degradation of measurement accuracy.

- For inspections tasks on (semi-) manufactured products, short cycle times are demanded for economic reasons. As a consequence CMMs are expected to operate with higher speed. Due to the resulting higher accelerations the effects of dynamic errors will also be increased.

- The increasing need for certification of products results in more attention for traceability of measurement results and their level of confidence. Thus sufficient knowledge about systematic and random errors, including dynamic errors, is necessary.

From the trends mentioned here, it is obvious 
that high accuracy as well as high speed is demanded. However, the demands for high measurement accuracy are conflicting with the wish for higher operating speed and shop floor conditions. Measurement speed of CMMs is often kept very low [1] to avoid a degradation of the measurement accuracy by dynamic errors. An alternative for the restriction of measurement speed is to obtain sufficient knowledge of all the dynamic errors and to apply software error compensation for these errors. The method of software compensation has been applied successfully by several researchers for quasi-static geometric errors [2-7]. Until recently, little attention has been paid to dynamic errors of CMMs and possibilities for compensation. Research concerning the dynamic behaviour of CMMs has been focused on theoretical and experimental methods for identifying the vibration modes of CMMs in order to improve CMM design. At the Precision Engineering section of the Eindhoven University of Technology (TUE), CMM research is now concentrated on fast probing and dynamic errors. The first contributions to this subject were focused on the identification of vibration modes [8] and the estimation of the vibration amplitudes. Recently, a research project has been finished concerning the dynamic errors of CMMs $[9,10]$. The main goal of this project was to investigate the possibilities for software error compensation of dynamic errors of CMMs due to fast probing and the practical implementation of a compensation method on a CMM. This paper describes the approach and presents the results of the experiments on an existing CMM.

\section{Dynamic errors of CMMS due to fast probing}

When referring to fast probing as opposed to normal probing, we not only mean a higher CMM speed, but more generally a reduction of the total cycle time of a measuring task. There are many factors that influence the cycle time of a measuring task and that have to be seen in relation to the measuring accuracy. These factors include: traverse and measuring speed, acceleration/deceleration, and approach distance. The relation between factors and the (dynamic) accuracy depends on the measuring task itself (measuring dimensions or profiles), the collection of measuring points (single points or scanning) and the type of probe used (touch trigger or measuring probe). In the case of taking single measuring points using a touch trigger probe, the same pattern of motion has to be followed each time in order to ensure proper probing (i.e. a well defined constant measuring speed at the time of contact). This particular pattern of motion greatly affects the cycle time of the measurement task as well as the accuracy. In the scheme shown in Fig. 1, the motion is described, indicating the acceleration, speed and position error of the probe versus time. During the speed changes, the inertial forces will cause dynamic position errors and, if probing, measurement errors. In order to avoid unacceptable dynamic errors, some settling time between decelerating and probing is necessary to allow the vibrations to settle. However, this is not always possible in practice. In the case of short approach distances the CMM will still be in the course of acceleration when contacting the measuring object. Especially in the case of small measuring elements, approach distances can often be very short and
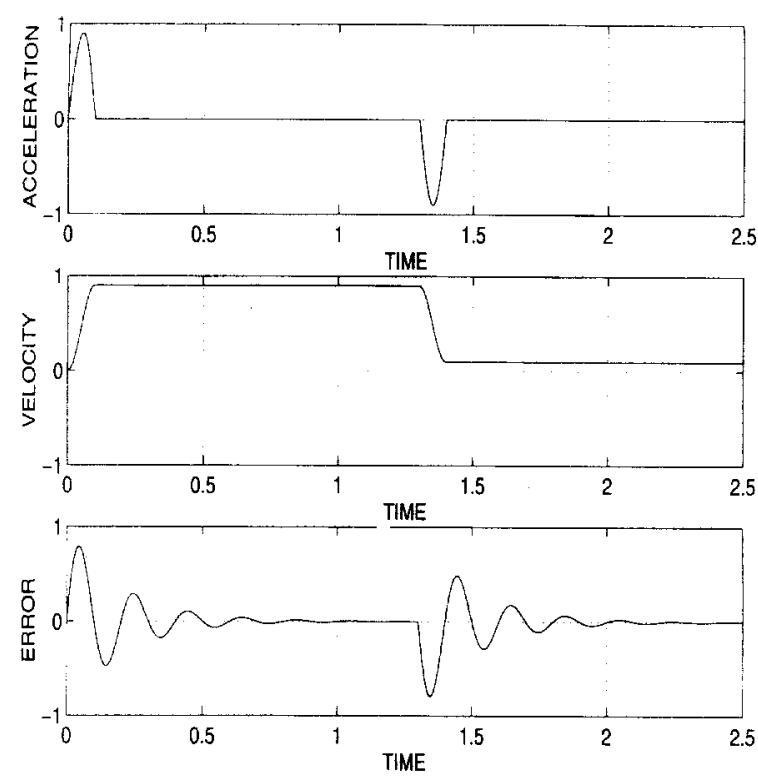

Fig. 1. Example of motion pattern during a measuring task (with normalised quantities). Top, axis acceleration; middle, axis velocity; bottom, probing error. 
thus the CMM is likely to be subjected to accelerations during the time of probing. Compared with touch-trigger probes, a measuring probe can sample multiple measuring points without renewed contact. The CMM only has to keep the tracking error within the range of the probe measuring system. This makes measuring probes very suitable for scanning and thus profile measurements. During high speed scanning of a profile nonlinear movements are generally required. As a result the CMM will be subjected to dynamic errors due to axes accelerations and drive induced vibrations. The effects of dynamic errors on the CMM's probe itself will not be discussed here. Details of such effects are described by van Vliet [11].

Regardless which task is performed and which type of probe is being used, the cycle time of a measuring task is influenced by the dynamic behaviour of a CMM's mechanical structure. The CMM's sensitivity for dynamic errors strongly depends on its structural loop. The structural loop is the part of the mechanical structure that comprises all the components that together define the position of the probe relative to the workpiece. Deformations of the structural loop e.g. due to driving forces and moving loads that cause (dynamic) errors with respect to the probe position, will inevitably affect the measuring accuracy. This is illustrated by an example of measurements on an existing CMM. In Fig. 2 a rotation error of a gantry type CMM is depicted. This error is caused by deceleration of the CMM before reaching a certain position. The maximum rotational error is over 5 arcsec, which yields translation errors at probe position of $25 \mathrm{~mm}$ for an Abbe offset of $1 \mathrm{~m}$.

When shorter cycle times of measurements are demanded, eventually higher speeds and thus accelerations during probing time cannot be avoided. This means that dynamic errors due to axis accelerations have to be accepted to some degree. In order to maintain an acceptable accuracy at probe position, estimation of these dynamic errors at the time of probing is necessary. For CMMs, exact knowledge of the (probe) position is sufficient in contrast to machine tools, where the programmed position has to be reached exactly. By applying compensation for position errors of the probe, in principle, time consuming position control is unnecessary. It is furthermore advantageous that, in principle, compensation for dynamic errors (based on the use of sensors) can also be applied to manual CMMs. These are very prone to dynamic errors since probing on a manual CMM is often performed in a rather uncontrolled way. The approach adopted here to achieve error compensation is of a combined analytical and empirical nature and contains the following steps:

- Describing the CMM structure with a kinematic model. With this model the degrees of freedom
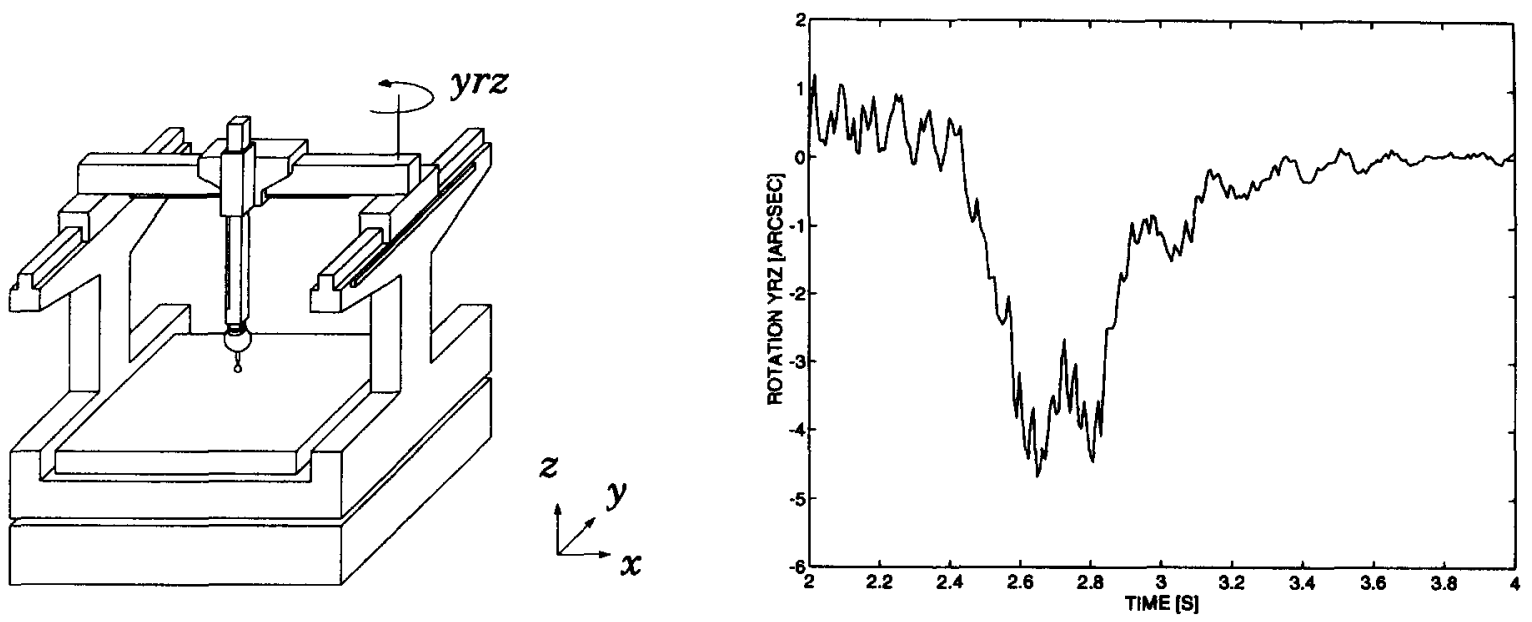

Fig. 2. Rotation error (right) caused by inertial effects on a gantry type CMM (left). 
of the CMM are described. Dynamic errors in the structural loop of the CMM have to be expressed in errors into these degrees of freedom (the parametric errors).

- Analysing the dynamic behaviour of the CMM in order to identify the significant deformations. Based on the results suitable sensors can be implemented on the CMM for measuring these significant errors on-line.

- Based on the measured values and the modelling of the relations between errors, the other relevant errors that are not measured can be estimated. The parametric errors are a combined effect of measured and estimated errors.

- Using the kinematic model for calculating the effect of the parametric errors on the probe position. The calculated error values at probe position during a certain measurement task are used for compensation of the measurement result.

It is important to realise that the modelling and analysis of the dynamic behaviour are not dependent on a particular CMM, but only on the type of CMM. This means that the error modelling and analysis of the dynamic behaviour have to be carried out only once for a certain type of CMM. The results can be used for all CMMs of the same type. This is important with respect to the efficiency of the proposed method. In general, differences between the actual machine parameters (e.g. stiffness values) that are used for estimating deformations based on the sensor measurements, will be small for different CMMs of the same type. However, in order to obtain a high accuracy and reliability of the method it is sensible to identify these parameters for each individual CMM. The modelling with respect to the dynamic errors and their effects on the probe position will be discussed next.

\section{CMM error modelling}

The main task here is the estimation of the exact probe position of a CMM each time a measurement is taken. The main errors that affect the structural loop of a CMM are geometric and thermal errors, errors due to mechanical loads and dynamic errors. For assessment of all these errors the same modelling approach can be used (see also Ref. [5]). In this way a modular compensation system is obtained. Depending on the circumstances, the various error sources will have more or less influence on the measuring accuracy. Taking into account the significance of the error sources and economical considerations, only compensation for some of the errors will be desirable. In the parametric modelling approach, the machine's errors are described as an analytical synthesis of errors introduced in the structural loop components. The basis of this approach is the kinematic error model. This model relates the errors in the relative location of the probe position to errors in the geometry of consecutive structural loop segments. The latter so-called parametric errors describe the combined effect of the various error sources on the geometry of the structural loop components that constitute such a segment, including the joints. In general, the parametric errors of a CMM are so small that the parametric errors of the different segments do not affect each other seriously.

For machines consisting of only prismatic joints in a Cartesian configuration, like most CMMs, a convenient vectorial notation can be used to describe the kinematic model. The error at the probe tip is defined as the difference between the actual probe tip position $p$ and the nominal position $d$ (see also the C-type CMM depicted in Fig. 3):

$e=-\underline{d}$

The errors related to axis $i$ of the CMM are described by two vectors containing the parametric translation and rotation errors of the axis' carriage:

$t_{i}=\left(\begin{array}{c}\text { it } x \\ \text { ity } \\ \text { itz }\end{array}\right)$ and $\underline{r}_{i}=\left(\begin{array}{c}\text { ir } x \\ \text { iry } \\ \text { irz }\end{array}\right)$

The error notation is according to the VDI-2617 guideline [12]. The first character denotes the axis of motion, the second the error type and the third the direction of the error or the axis of rotation. The angular errors are defined as rotations about mutually perpendicular axes. The propagation to 


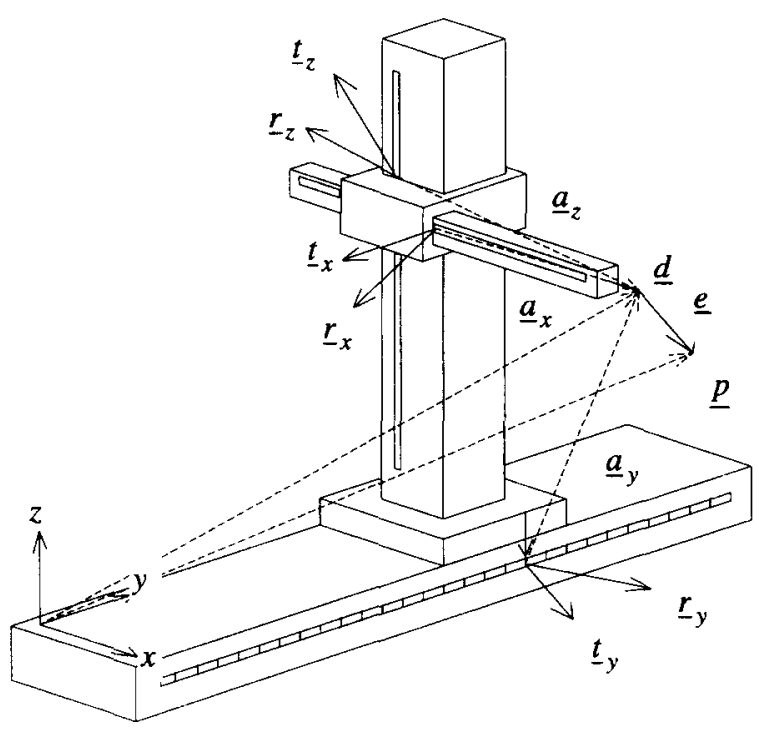

Fig. 3. Definition of the various vectors used in the kinematic model of a CMM.

the probe position of the errors described by vectors, can be expressed as:

$\underline{e}_{i}=\underline{t}_{i}+\underline{r}_{i} \times \underline{a}_{i}$

The vector $\underline{a}_{i}$ represents the effective arm between the scale at axis $i$ and the probe tip, including the probe itself. Thus if the probe configuration is changed, the arm vector has to be adapted. Combining the contributions of all axes yields the total position error at the probe tip:

$\underline{e}=\sum_{i} \underline{e}_{i}$

Although a rigid-body kinematic model is used for the error propagation of the parametric errors, the components of a CMM structure (joint and link elements) are here considered as flexible elements. A certain parametric error, belonging to one of the three CMM axes, will be a combination of element deformations. Thus, for example, the parametric rotation error irj can be written as a summation of the deformations of several components $c$ of axis $i$ :

$\operatorname{irj}=\sum_{c=1}^{n_{i}} \epsilon_{j, c}$

In our approach the identification of the paramet- ric errors of a CMM is based on their measurement with additional sensors mounted on the CMM. In practice, the deformations of the joints will often give the largest contributions to the error at probe position. A good possibility for measuring these deformations is to attach displacement sensors to the respective carriage. The sensors measure the relative displacements of the carriage sides perpendicular to the guideway. From the combined measurements of the two sensors on both sides of the carriage, one of the carriage's rotations and one of the translations can be found. However, the use of sensors has to be limited. Their number will be a pay-off between accuracy and economical reasons. Our approach is aimed at the use of a minimum number of extra sensors, but with sufficient accuracy. This can be achieved by relating the measured deformations to the other relevant deformations.

In Fig. 4 the $y$-carriage and guideway of a CMM are depicted schematically. Let us consider the rotation errors about the $z$-axis that can be related to the $y$-axis. The components that can contribute to the parametric rotation error $y r z$ are: the $y$ carriage, its bearing system, its support, and the $x$-guideway. Using Eq. (5) the parametric error $y r z$ can be expressed as:

$$
\begin{aligned}
y r z= & \sum_{c=1}^{n_{y}} \epsilon_{z, c}={ }_{y} \epsilon_{z, \text { carriage }}+{ }_{y} \epsilon_{z, \text { bearings }}+{ }_{y} \epsilon_{z, \text { support }} \\
& +{ }_{y} \epsilon_{z, x} \text { - guideway }
\end{aligned}
$$

First we consider only the first three components that together form the $y$-carriage joint (assuming no significant bending of the $x$-guideway). For convenience, subscripts $y$ and $z$ are further omitted. The $y$-carriage joint can be represented schematically by a mass-spring system, as shown in Fig. 5(a). In general, the relationship between the various deformations can be described by sets of differential equations. However, for the joint, the mass moment of inertia of the traverse (including the components of the $x$ - and $z$-axis) will be much larger than the moments of inertia of the other components, that are located close to the axis of rotation. In this case a simplified model can be obtained by neglecting the moments of inertia of the carriage and the support. Furthermore, it is 

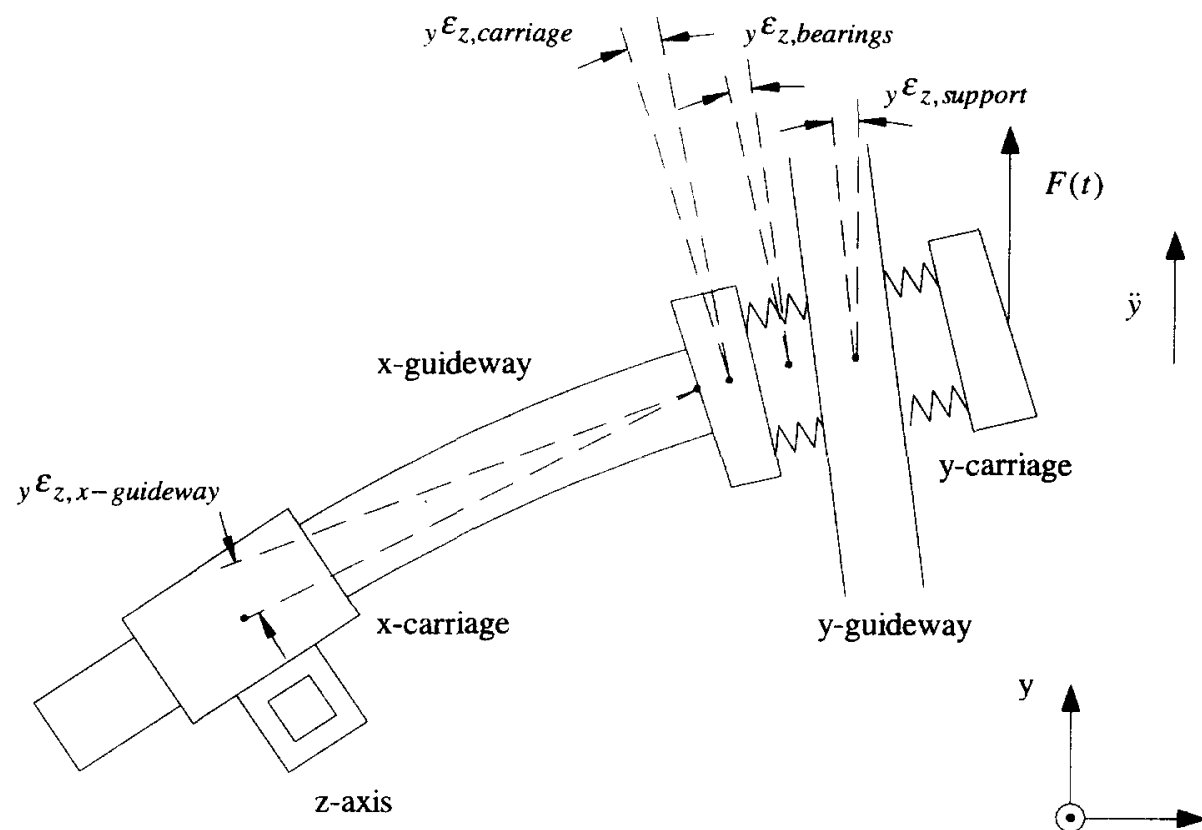

Fig. 4. The rotational deformations of the $y$-axis of a CMM due to $y$-motion.

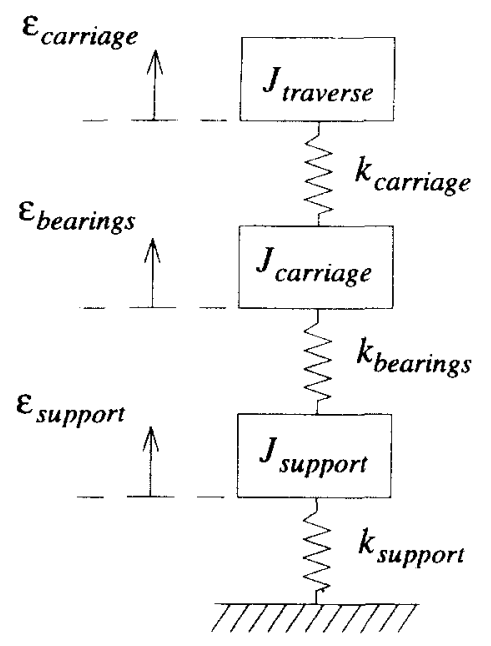

a.

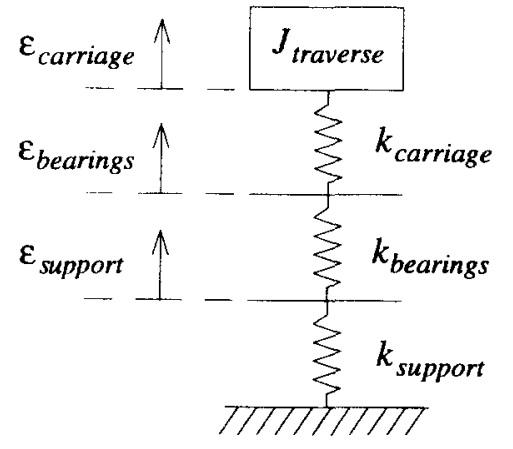

b.

Fig. 5. Mass-spring systems representing the components of the y-carriage joint. (a) System with components that all have inertial mass. (b) Simplified system with only the last element having inertial mass.

assumed that the stiffness of the bearing system is constant over the frequency range of interest and can be represented by its static stiffness. The frequencies of interest are the lower frequencies of the CMM induced by motion of the axis. In this model the system is represented by a series of springs and a single mass moment of inertia (see Fig. 5(b)). Due to the absence of the inertia 
moments between the springs, the same moment $M_{\text {traverse }}$ is acting on each of the springs. Thus for $J_{\text {carriage }}$ and $J_{\text {support }}$ both « $J_{\text {traverse, }}$, we can write:

$M_{\text {support }}=M_{\text {bearings }}=M_{\text {carriage }}=M_{\text {traverse }}$

or

$k_{\text {support }} \epsilon_{\text {support }}=k_{\text {bearings }} \cdot \epsilon_{\text {bearings }}=k_{\text {carriage }} \cdot \epsilon_{\text {carriage }}$

Where the rotational stiffness parameters $k_{i}$ are assumed constant. Using this relationship we can write for the parametric error of the joint:

$y r z=\left(1+\frac{k_{\text {bearings }}}{k_{\text {carriage }}}+\frac{k_{\text {bearings }}}{k_{\text {support }}}\right) \cdot \epsilon_{\text {bearings }}$

If the bearing rotations (the rotations between the bottom of the carriage and the guideway) are measured and the stiffness ratios are known, the parametric error for the joint can be estimated using this relationship. In general:

$i r j=\left(\sum_{c=1}^{n} \frac{k_{m}}{k_{c}}\right)_{i} \epsilon_{j, m}$

where ${ }_{i} \epsilon_{j, m}$ denotes the measured rotation error of component $m$ about the $j$-axis during motion in the $i$-direction, and $k_{m} / k_{c}$ the stiffness ratio between the measured component $m$ and component $c$. Link deformations will not be discussed in detail here (see Ref. [10]), they can be dealt with similarly to the joint errors, assuming that the displacement field of the link in the dynamic situation is similar to the static deformation and that the bending rotation is in phase with the carriage rotation. The deformations can be calculated from the accelerations which in turn can be estimated from the sensor measurements. In general, link deformations can be more complex, but the deformation assumed here will cause the largest errors. Furthermore, the link deformations of CMMs are often small compared with joint deformations (so also next paragraph). Therefore it is reasonable to expect that the contributions of the more complex deformations of the links are negligible compared with the joint deformations and the assumed bending deformation of the link. If expressions are found for all relevant parametric errors their values can be calculated based on on-line measurements by the sensors. Using the kinematic model the probe error can be calculated for each position at any moment.

Translation errors can be modelled in the same way as the rotation errors. In general, the influence of these translation errors is small, because the structural loop is not very sensitive in the direction of the relevant translation errors, i.e. the errors perpendicular to the guideways. The stiffness of the various elements of the CMM's axes with respect to these directions is relatively high, and in general only the bearing compliance will significantly contribute to the translation errors. The stiffness in the direction of movement of an axis can be much lower, in contrast to the stiffness in the other two directions. This will only cause linearity errors that are measured directly by the scales. Thus these translation errors will not affect the measuring accuracy. Only if an axis is weakly supported, deformations of this support, e.g. bending, can contribute to the translation errors.

\section{Compensation of a CMM}

The described approach has been applied to an existing CMM at the TUE. The dynamic behaviour of this CMM was investigated and can be summarised as follows:

- Significant dynamic errors are induced by motion of the $x$ - and $y$-axis: $y r z, y r x, x r x, x r y$. Translation errors are insignificant, except for bending of the $y$-guideway support ( $y t y)$.

- The behaviour of the $y$-axis is rather dominant. The low stiffness of its drive causes translational vibrations, and this also induces rotational vibrations due to inertia effects.

- The error levels are affected by the accelerations that are set by the commanded velocities or type of control. Especially during joystick control are large errors found.

- Errors depend on carriage positions. This changes the effective arm of the acceleration forces on a carriage, causing changes in the moment applied to another carriage.

For the investigated CMM (see Fig. 2) the kinematic model depicted in Fig. 6 was derived. The 


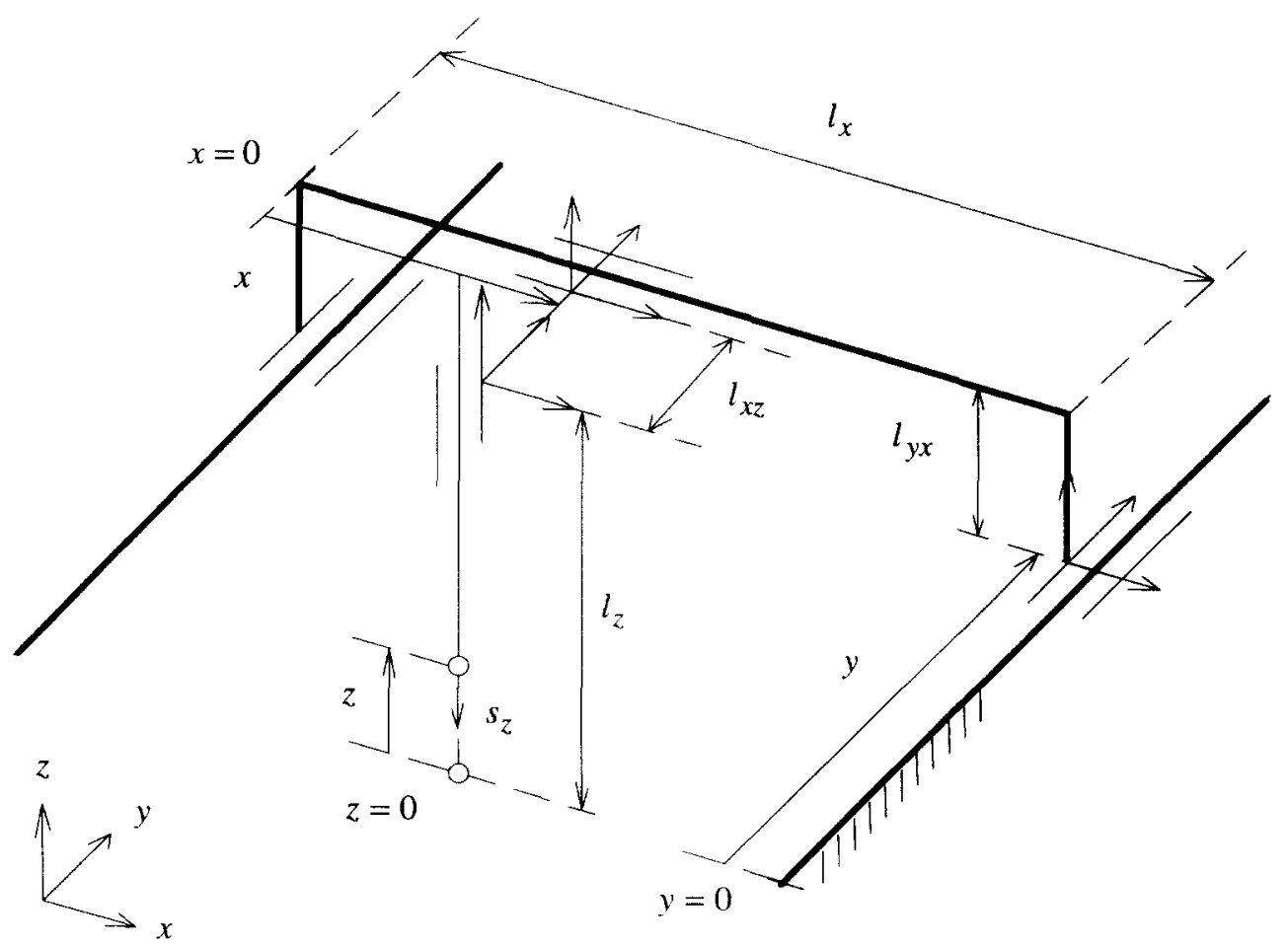

Fig. 6. Kinematic model of the investigated CMM.

figure shows the three coordinate frames and the dimensions that are necessary for calculating the length of the effective arms of rotation. These can be expressed by:

$\underline{a}_{x}=\left(\begin{array}{c}0 \\ -l_{x z} \\ z-l_{z}-s_{z}\end{array}\right) \underline{a}_{y}=\left(\begin{array}{c}x-l_{x} \\ l_{x z} \\ z+l_{y x}-l_{z}-s_{z}\end{array}\right)$

$\underline{a}_{z}=\left(\begin{array}{c}0 \\ 0 \\ z-l_{z}-s_{z}\end{array}\right)$

With these vectors the propagation of the parametric errors to the probe position can be calculated using Eq. (3). The parametric errors can be obtained from on-line sensor measurements. The sensors used are inductive displacement sensors, measuring the carriage motion perpendicular to the guideway at the bearing positions due to their deflections. Using a set of two sensors, the carriage rotation about one axis and the translation in one direction can be measured. The expressions between the relevant parametric errors and the measured (bearing) deformations are for the CMM used:

$$
\begin{aligned}
y r z & =\left(1+\frac{y k_{z, \text { bearings }}}{y k_{z, \text { carriage }}}+\frac{y k_{z, \text { bearings }}}{y k_{z, \text { support }}}\right) \cdot{ }_{y} \epsilon_{z, \text { bearings }} \\
& \approx 2.2 \cdot{ }_{y} e_{z, \text { bearings }} \\
y r x & =\left(1+\frac{y k_{x, \text { bearings }}}{y k_{x, \text { carriage }}}+\frac{y k_{x, \text { bearings }}}{y k_{x, \text { support }}}\right) \cdot{ }_{y} \epsilon_{x, \text { bearings }} \\
& \approx 2.0 \cdot{ }_{y} \epsilon_{x, \text { bearings }} \\
x r x & =\left(1+\frac{x k_{x, \text { bearings }}}{x k_{x, \text { carriage }}}\right) \cdot{ }_{x} \epsilon_{x, \text { bearings }} \approx 1.9 \cdot{ }_{x} \epsilon_{x, \text { bearings }} \\
x r y & =\left(1+\frac{x k_{y, \text { bearings }}}{x k_{y, \text { carriage }}}\right) \cdot{ }_{x} \epsilon_{y, \text { bearings }} \approx 1.7 \cdot{ }_{x} \epsilon_{y, \text { bearings }}
\end{aligned}
$$




$$
\begin{aligned}
y t y & =f_{y y}(x) \cdot \frac{y k_{z, \text { bearings }}}{y k_{y, \text { support }}} \cdot{ }_{y} \epsilon_{z, \text { bearings }} \\
& \approx 0.5 \cdot f_{y y}(x) \cdot{ }_{y} \epsilon_{z, \text { bearings }}
\end{aligned}
$$

The stiffness ratios are obtained from off-line measurements. Measurements show that the ratios found are reasonably constant for different carriage positions. Note, however, that the $y t y$ error, describing the motion of the support, is dependent on the $x$-carriage position. The factor $f_{y y}(x)$, denotes the relationship between the moment due to the $y r z$-rotation, applied to the $y$-carriage and the reaction force of the carriage on the support. This allows the estimation of the support motion from the measured bearing rotation (see Ref. [10]). In fact there is also a reaction force and resulting support motion due to the $y r x$-rotation. This effect can be estimated from the $y r x$-rotation but is insignificant in this case.

Using the kinematic model the probe error of the investigated CMM can be expressed into the above mentioned significant parametric errors. We can write for the components of the error $\underline{e}$ at probe position (where $e=\left(e_{x}, e_{y}, e_{z}\right)$ ):

$e_{x}=y r z \cdot l_{x z}+x r y \cdot\left(z-l_{z}-s_{z}\right)$

$$
\begin{aligned}
& e_{y}=-x r x \cdot\left(z-l_{z}-s_{z}\right)+y t y+y r z \cdot\left(x-l_{x}\right) \\
& \quad-y r x \cdot\left(z+l_{y x}-l_{z}-s_{z}\right) \\
& e_{z} \approx 0
\end{aligned}
$$

In Fig. 7 the investigated CMM is depicted together with the implemented sensors. Two sets of sensors are attached to the $y$-carriage and also two sets to the $x$-carriage (only six sensors could be used simultaneously, since only six channels at the amplifier system were available). These sensors are used to identify the rotation errors expressed by Eqs (12)-(15). Note that the four sets of sensors can also be used to identify four translation errors as well. However these are not significant. As an example of the error that can be expected at probe position during motion, an estimation of the error in $y$-direction will be given here. This error is the most significant error for this CMM, and there are significant contributions of four parametric errors. Three of these errors can be measured by the sensors. The support motion, expressed by the parametric error $y t y$, is estimated on basis of the sensor readings for the error $y r z$. In this example the CMM was programmed to move with traverse speed ( $70 \mathrm{~mm} \mathrm{~s}^{-1}$ ) along the $y$-axis to a certain position.

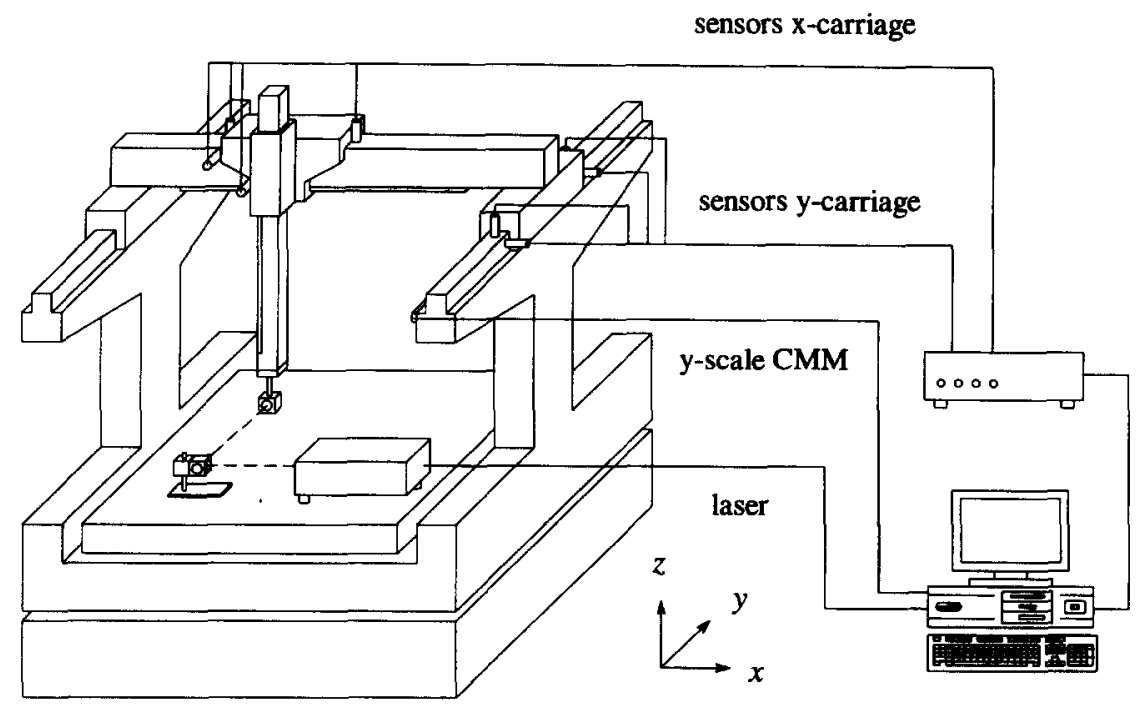

Fig. 7. The investigated CMM with the implemented sensors attached to the $y$-and $x$-carriages. 

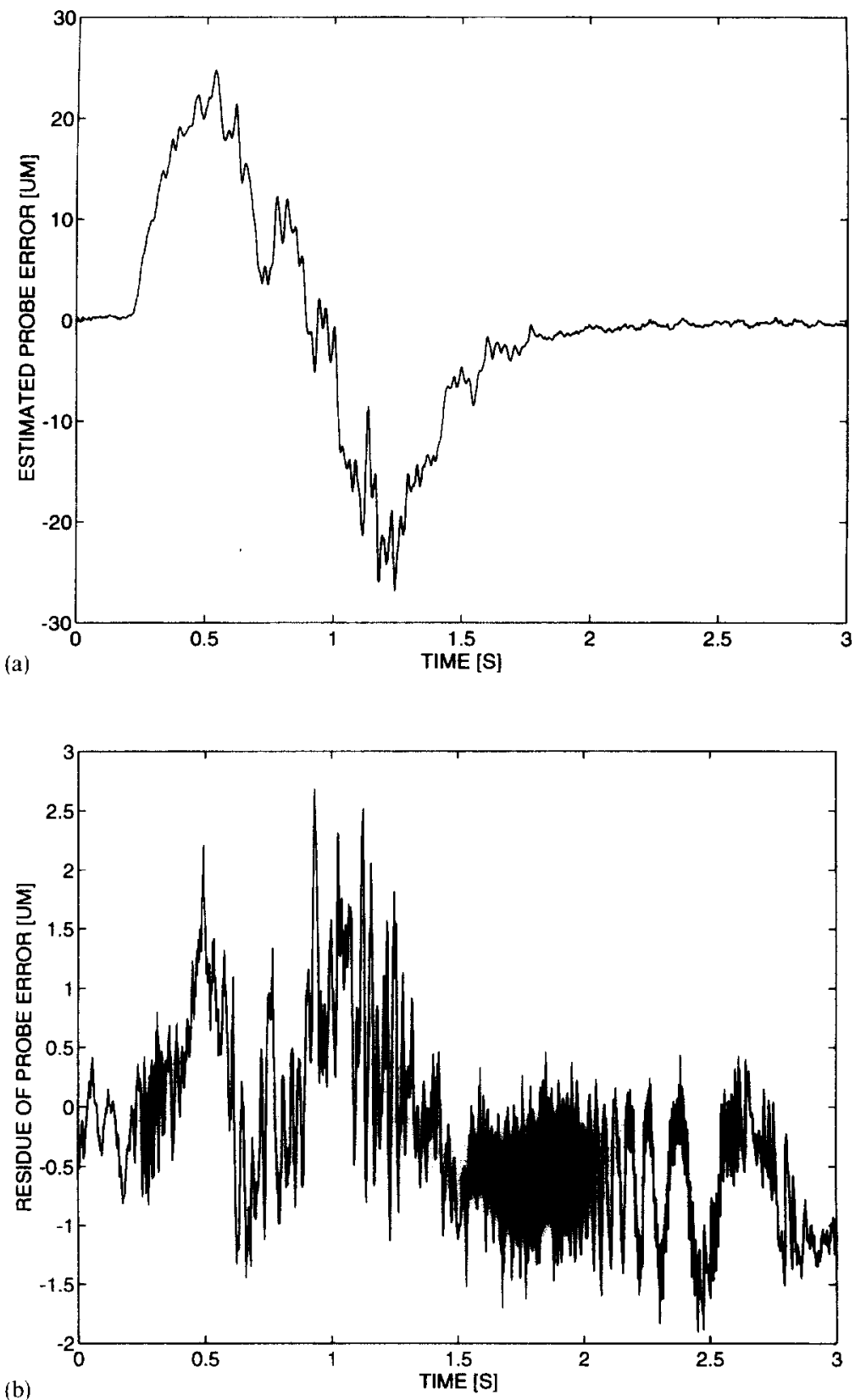

Fig. 8. (a) The dynamic error in the $y$-direction at the probe position during $y$-axis motion, calculated on the basis of the parametric errors. (b)The difference between the measured and estimated dynamic error in the $y$-direction at probe position during $y$-axis motion. The CMM is accelerating to and decelerating from traverse speed.

The $x$-carriage and the $z$-pinole were both in their zero positions, resulting in maximum effective arms for the respective parametric rotation errors.
Based on the sensor data and Eqs (12)-(16) the parametric errors that occurred were found. Using Eq. (18) the error at probe position was calcu- 
lated. This error is depicted in Fig. 8(a). The graph shows clearly that the dynamic error at the probe position is quite large compared with the static inaccuracy (maximum $25 \mu \mathrm{m}$ dynamically versus less than $4 \mu \mathrm{m}$ statically). This means that if measurements are made when the CMM is still subjected to accelerations, compensation of the measuring result is necessary in order to obtain a
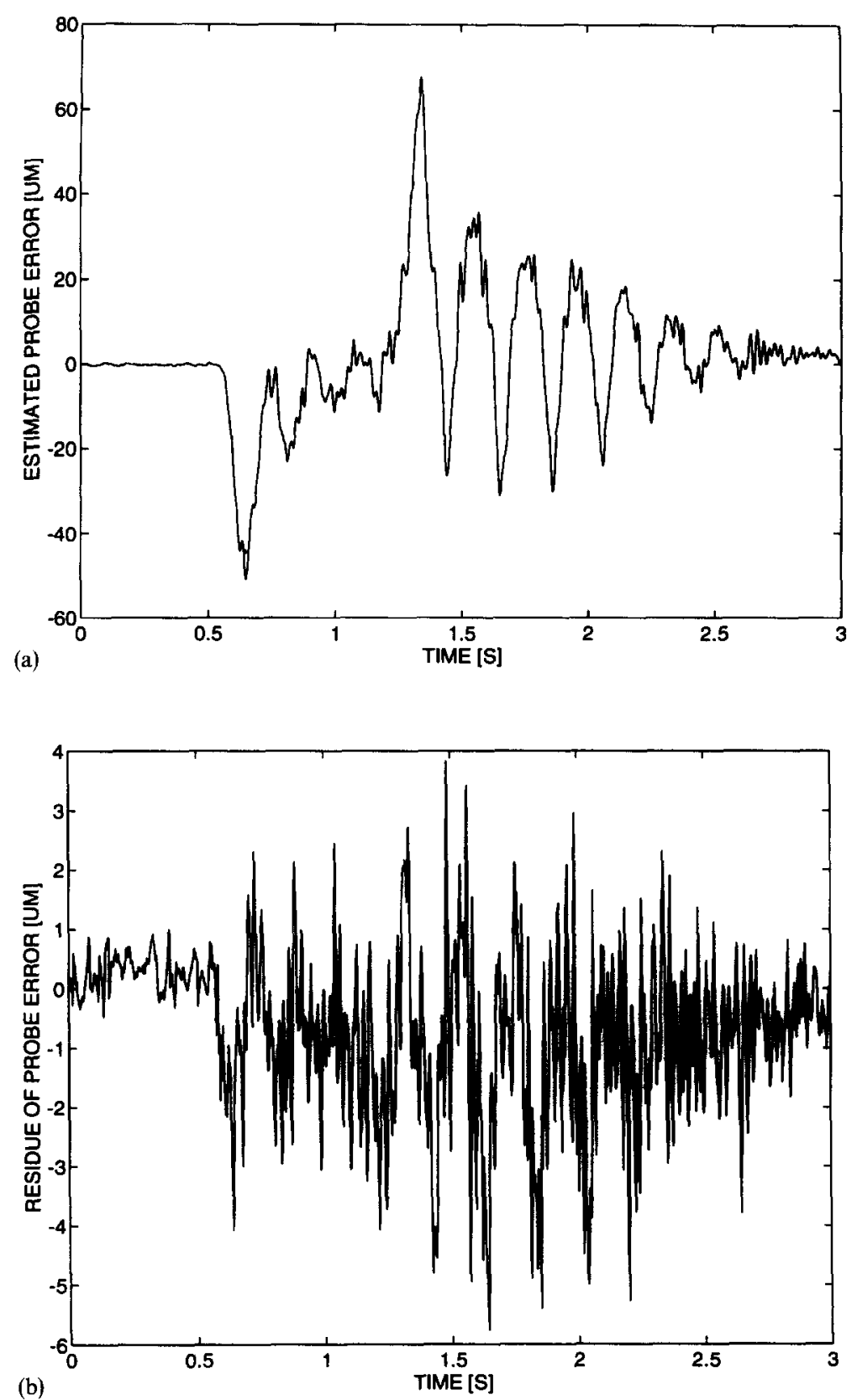

Fig. 9. (a) The estimated dynamic error in the $y$-direction at probe position during $y$-axis motion. The CMM is decelerating suddenly. (b) The difference between the measured and estimated dynamic error in the $y$-direction at probe position during $y$-axis motion. 
sufficient accuracy (i.e. comparable with the static inaccuracy).

Using a laser interferometer with linear optics the displacement at probe position was also measured directly. In order to obtain the error at probe position (i.e. the difference with respect to the scale readings) the CMM's $y$-scale readings had to be read simultaneously. In Fig. 8(b) the difference between the estimated and the measured error is given. This residue is at maximum $2.5 \mu \mathrm{m}$, which is acceptable considering the static accuracy of approximately equal magnitude (better than $4 \mu \mathrm{m})$. Compared with the maximum error found, the residue is approximately $10 \%$. Thus in this case the dynamic error that occurs can be compensated for by $90 \%$, using the compensation method based on position sensors. In the previous example an estimation of a dynamic error was given that showed mainly quasi-static effects. In Fig. 9(a) the estimated error for a vibration, that occurs after suddenly decelerating, is shown. The difference with the actual error is shown in Fig. 9(b). In this case the CMM was joystick controlled. Due to the larger accelerations, the error at probe position is higher than in the previous case. Also the residue is higher in this case, approximately $5 \mu \mathrm{m}$, but the relative error is again about $10 \%$. From these examples it is clear that at least for one-axis motion of this CMM, the dynamic errors can be compensated for very well.

\section{Conclusions}

This paper describes a method for the estimation of dynamic errors that occur at a CMM's probe position in case of higher probing speeds. The method uses additional sensors attached to the carriages of a CMM, for on-line measurement of the deformations caused by inertia effects due to axes motion. Beam vibrations are not considered. Relationships are modelled between the measured deformations and the significant parametric errors. A kinematic model is used to calculate their effect on the probe position. The developed method has been applied to an existing CMM in order to verify its performance in practice. Based on the sensor readings and the developed models, the time history of the dynamic error at probe position could be calculated. The estimated probe error showed good resemblance with the actual error. The results of the verification measurements show that, at least for single axis motion, by compensating for dynamic errors of CMMs, still a high accuracy can be obtained when the CMM is subjected to accelerations. In this way fast probing is possible without significant degradation of measurement accuracy.

Further experiments will be performed in order to determine the accuracy improvement that can be obtained for multi-axes motion. In order to reduce the costs of compensation the number of sensors has to be reduced and other type of sensors than the sensors used during our investigations have to be used. Capacitance and pneumatic displacement sensors are interesting options. Future research is aimed at their implementation on a CMM.

\section{Acknowledgements}

The authors acknowledge Renishaw Transducer Systems Ltd for supplying a fast laser interferometer system. They also wish to thank Ryoshu Furutani of Tokyo Denki University, Japan and Leon Levasier for their help with interfacing the measuring equipment.

\section{References}

1. Phillips, S. D., Borchardt, B. and Caskey, G., Measurement uncertainty considerations for coordinate measuring machines. NIST, Gaithersburg, 1993.

2. Busch, K., Kunzmann, H. and Wäldele, F., Numerical error correction of a coordinate measuring machine. Proceedings of the International Symposium on Metrology and Quality Control in Production, 1984, pp. 278-282.

3. Kruth, J, P., Vanherck, P. and De Jonge, L., A selfcalibration method and software error correction for three dimensional coordinate measuring machines. Proceedings of the International Symposium on Metrology and Quality Control in Production, 1992, pp. 100-115.

4. Schellekens, P. H. J. et al., Development of methods for the numerical error correction of machine tools. Final report, BCR, 3320/1/0/160/89/8،BCR`NL, Brussels, 1993. 
5. Soons, J. A., Accuracy analysis of multi-axis machines. $\mathrm{PhD}$ thesis, Eindhoven University of Technology, 1993.

6. Teewsen, J. W. M. C., Soons, J. A. and Schellekens, P. H. J., A general method for error description of CMMs using polynomial fitting procedures. Annals of the CIRP, 1989, 38, 505-510.

7. Zhang, G., Error compensation of coordinate measuring machines. Annals of the CIRP, 1985, 34, 445-448.

8. de Nijs, J. F. C., Lammers, M. G. M., Schellekens, P. H. J. and van der Wolf, A. C. H., Modelling of a coordinate measuring machine for analysis of its dynamic behaviour. Annals of the CIRP, 1988, 37, 507-510.
9. Weekers, W. G. and Schellekens, P. H. J., Assessment of dynamic errors of CMMs for fast probing. Annals of the CIRP, 1995, 44, 469-474.

10. Weekers, W. G., Compensation for dynamic errors of coordinate measuring machines. PhD thesis, Eindhoven University of Technology, 1996.

11. van Vliet, W. P., Development of a fast mechanical probe for coordinate measuring machines. Ph.D. thesis, Eindhoven University of Technology, 1996.

12. VDI/VDE 2617, Accuracy of coordinate measuring machines; Characteristic parameters and their checking. VDI-Verlag, Düsseldorf, Germany, 1991. 